Meta

Journal des traducteurs

Translators' Journal

\title{
La pratique québécoise de la normalisation terminologique
}

\section{Louis-Jean Rousseau}

Volume 36, numéro 1, mars 1991

La terminologie dans le monde : orientations et recherches

URI : https://id.erudit.org/iderudit/004005ar

Aller au sommaire du numéro

Éditeur(s)

Les Presses de l'Université de Montréal

ISSN

0026-0452 (imprimé)

Découvrir la revue

Citer cet article

Rousseau, L.-J. (1991). La pratique québécoise de la normalisation

terminologique. Meta, 36(1), 225-230. d'utilisation que vous pouvez consulter en ligne.

https://apropos.erudit.org/fr/usagers/politique-dutilisation/ 


\section{LA PRATIQUE QUÉBÉCOISE DE LA NORMALISATION TERMINOLOGIQUE}

LOUIS-JEAN ROUSSEAU

Office de la langue française, Québec, Canada

\section{LA NOTION DE NORMALISATION}

La normalisation dont il sera ici question est d'abord et avant tout la normalisation officielle de la terminologie, que l'ISO définit ainsi : «officialisation d'une terminologie par un organisme qui fait autorité ${ }_{\gg}$.

Cependant cette normalisation doit être replacée dans un ensemble plus grand de pratiques normalisatrices dont le volume et l'importance dépassent souvent le produit de la normalisation officielle.

Le premier type de normalisation qui vient ainsi à l'esprit est la normalisation technique. Mais cette normalisation technique dont l'incidence terminologique est parfois considérable s'avère impossible sans une normalisation notionnelle, qui entraîne à son tour une normalisation terminologique ou, à tout le moins, qui suggère par l'exemple un usage terminologique particulier dans une norme technique. Ce type de normalisation est effectué par des organismes comme l'ISO, l'AFNOR, le BNQ, etc.

Il existe un autre type de normalisation plus insidieux, car il ne se présente pas comme tel. Il s'agit de la normalisation terminologique effectuée par le biais des textes officiels : lois, règlements, décrets, arrêtés, circulaires, etc.

Le discours juridique comporte cette particularité de créer des réalités et, par la même occasion, de circonscrire des notions et de les dénommer. La loi crée des termes ou reprend des termes existants en les sanctionnant officiellement. Cette pratique crée et impose des usages terminologiques.

En effet, en choisissant d'utiliser tel terme pour désigner une notion, le législateur impose un usage terminologique qui sera suivi par toute l'Administration puis, dans une large mesure, par les administrés, qui n'ont alors guère le choix. Il semble que le caractère impératif de la loi s'étende dans les faits à la terminologie utilisée 2 .

Il est enfin un autre type de normalisation qui, tout en s'effectuant en dehors des voies officielles, peut avoir une importance considérable. C'est la normalisation terminologique conduite à l'intérieur de divers corps professionnels, au plan national ou international. Citons comme exemple les travaux terminologiques de l'Union internationale de l'industrie du gaz, ceux de la Conférence mondiale de l'énergie ou ceux de l'Organisation mondiale de la santé. Le même type de normalisation peut également s'effectuer à l'échelle de l'entreprise ou d'un groupe d'entreprises cuvrant dans le même secteur.

Bien que n'ayant aucun caractère officiel, cette normalisation dépasse probablement en volume et en impact réel tout ce que la normalisation officielle a pu produire.

Certains diront qu'il ne s'agit plus de normalisation mais de diffusion ou, pour reprendre l'expression de $\mathrm{R}$. Kocourek, d' «autorégulation terminologique» ${ }^{3}$. Mais, si l'on tient compte des résultats, on peut assimiler ce processus à la normalisation dans son sens étendu. 
La sociolinguistique nous apprend que les usages linguistiques sont, comme les autres facettes du comportement humain, influencés, voire conditionnés par un certain nombre de facteurs. En communication, personne n'est libre de créer son propre code s'il souhaite transmettre un message qui soit compris. La performance individuelle ne s'exerce qu'à l'intérieur de certaines balises fixées par le système linguistique. La normalisation terminologique dans ses diverses réalisations est un des facteurs qui tentent d'influencer la performance linguistique des sujets parlants. Mais contrairement aux autres agents de conditionnement de l'usage (publicité, médias, école, etc.), la normalisation terminologique est consciente et réfléchie. Elle vise à assurer la compréhension identique par les locuteurs des significations des notions que sous-tendent les termes, conduisant ainsi à la réalisation de la communication effective: les termes utilisés par l'émetteur sont compris et acceptés comme tels par l'interlocuteur.

\section{LA NORMALISATION DE LA TERMINOLOGIE À L'OFFICE DE LA LANGUE FRANÇAISE}

Avec l'adoption successive de plusieurs lois à caractère linguistique, le Québec s'est doté d'une politique d'aménagement linguistique qui porte à la fois sur le statut du français et sur le corpus du français parlé et écrit au Québec. Le mandat de normalisation que la Charte de la langue française a confié à l'Office s'inscrit dans cette perspective.

La Charte donne à l'Office le devoir de normaliser les termes et expressions qu'il approuve, et le pouvoir de créer des commissions de terminologie. Ces dernières doivent faire l'inventaire des lacunes terminologiques de l'Administration et dresser la liste des termes qu'elles préconisent. La Charte précise de plus la portée des décisions de l'Office en matière de normalisation. L'emploi des termes normalisés devient obligatoire dans les textes, les documents et l'affichage émanant de l'Administration, ainsi que dans les contrats auxquels elle est partie. De plus, ces termes normalisés doivent être utilisés dans les ouvrages d'enseignement, de formation ou de recherche publiés en français au Québec et approuvés par le ministre de l'Éducation ou par le ministre de l'Enseignement supérieur.

Ce mandat de normalisation peut sembler très vaste, compte tenu du volume des terminologies traitées à l'Office en réponse aux immenses besoins suscités par l'aménagement linguistique. Au départ, deux grandes questions se sont posées: faut-il tout normaliser? quels sont les critères à mettre en œuvre?

À la première question, l'Office a choisi de répondre par la négative, et ceci pour deux raisons principales. La première, c'est qu'il n'est pas nécessaire de tout normaliser, le consensus sur les usages à promouvoir étant la plupart du temps relativement facile à obtenir. La seconde, c'est que la normalisation systématique, compte tenu des exigences méthodologiques de la démarche normalisatrice, aurait demandé des ressources considérables. L'Office a donc choisi d'user avec prudence de son pouvoir de normalisation en n'intervenant que sur des cas problèmes ou sur des cas pour lesquels un avis officiel sollicité était justifié.

En effet, en dix ans de pratique, l'Office n'a émis que 1600 avis officiels ${ }^{4}$.

D'autre part, l'exercice du mandat de normalisation a nécessité, outre la mise en œuvre de moyens particuliers, la définition de principes d'intervention.

Au plan des moyens, l'Office s'est doté de sa propre commission de terminologie (la CTOLF), constituée de terminologues chevronnés représentant différents milieux tels l'Administration, les entreprises, les universités et les services linguistiques de l'Office.

Cette commission a été chargée de proposer à l'Office des principes de normalisation et de les mettre en œurre dans le traitement des demandes. 


\section{MODE DE FONCTIONNEMENT}

Pour la conduite de ses travaux, la CTOLF s'est dotée d'un mode de fonctionnement qui s'appuie sur des critères quant à l'acceptation de la demande et quant au statut d'officialisation (recommandation, pré-normalisation, normalisation). Ce mode de fonctionnement permet de donner une assise rigoureuse aux propositions terminologiques en assurant la consultation des usagers et en tentant d'établir des consensus qui tiennent compte de l'usage québécois et de l'usage international. La procédure d'adoption d'un avis comporte les étapes suivantes :

1) acceptation ou rejet de la demande;

2) préparation d'un dossier (exposé du problème, description des usages, consultation du milieu visé, proposition);

3) étude des dossiers par la CTOLF;

4) contre-expertise ou consultation élargie au besoin;

5) étude par l'Office;

6) publication d'un avis de recommandation ou d'un préavis de normalisation suivi d'une période de consultation d'un an.

Ces étapes peuvent sembler faire preuve de lourdeur mais, s'agissant de décisions terminologiques officielles, elles sont essentielles à l'exercice de choix raisonnés (et raisonnables).

\section{PRINCIPES D'INTERVENTION}

Tout d'abord vient la question de la nature des données à traiter. La nature terminologique du mandat de normalisation confié à l'Office ne fait pas de doute et la CTOLF s'en est généralement tenue à ces questions, à l'exception d'un petit nombre de sujets extra-terminologiques tels que l'accentuation des majuscules.

Toutefois le traitement par l'Office de termes appartenant à des domaines voisins de la vie quotidienne a parfois fait l'objet de critiques de la part de certains qui y ont vu une intervention normalisatrice sur la langue générale. Ces critiques ne tiennent pas compte des différences qui existent entre la terminologie et le lexique de la langue générale. Est-il besoin de rappeler que, alors que le mot appartient à l'univers de la signification et de la connotation, le terme est un nom, au sens de name ou de benennung, et: appartient à l'univers de la référence et de la dénomination. La confusion entre terme et mot vient du fait que ce sont souvent les mêmes formes qui sont en cause, bien que constituant deux objets différents. Ce qui caractérise le terme, c'est l'existence d'un rapport univoque entre une forme lexicale et une notion donnée qui représente l'objet à nommer. Par exemple, le terme chat en zoologie est un objet tout à fait distinct du mot chat en langue générale; la définition terminologique de la notion de chat situera l'animal dans l'ordre des carnivores et dans la famille des félidés, et énumérera les caractères qui permettent de différencier cette notion des notions voisines, alors que pour le mot chat, le lexicographe donnera une définition connotative; la valeur d'emploi du mot chat sera illustrée par des exemples permettant de dégager l'identité fonctionnelle du mot en discours, notamment par des relations d'opposition (ex.: se battre comme chiens et chats) ou des relations d'association (ex. : le chat mange la souris).

L'appartenance des termes à des domaines de la vie quotidienne n'en fait pas des unités de la langue générale pour autant, qu'il s'agisse d'alimentation, de vêtement ou de tout autre domaine de même nature.

Par ailleurs, le processus de normalisation n'est mis en œuvre que pour les cas où l'officialisation est jugée nécessaire. Dans le cas des demandes issues des commissions ministérielles, il est essentiel que ces demandes soient pertinentes par rapport à leurs domaines respectifs d'intervention. Pour ce faire, le choix des unités traitées par ces 
commissions fait l'objet d'ententes avec l'Office de la langue française, et il doit être strictement en rapport avec la langue dite institutionnelle, et plus spécialement avec les domaines de spécialité des ministères.

La normalisation terminologique doit s'appuyer sur une description de l'usage. C'est la seule façon de rendre cette démarche crédible et d'obtenir un consensus des usagers. Cependant, quand il s'agit de néologie - et c'est souvent le cas en situation de normalisation - il n'y a pas d'usage à constater. Par conséquent, le choix des termes à privilégier doit se fonder sur une démarche terminologique rigoureuse incluant des critères terminologiques tels que la conformité au système linguistique, la facilité de graphie et de prononciation, la systémicité, la dérivabilité, la motivation, l'exactitude, la concision, etc. À ces critères, il faut ajouter des critères d'acceptabilité sociolinguistiques qui ont trait à l'implantabilité des termes dans l'usage.

Une fois choisis les termes à privilégier, il faut déterminer le statut de la décision qui sera prise. À l'Office, il est prévu que certains termes peuvent être normalisés alors que d'autres ne seront que recommandés. Cette nuance a été apportée afin de donner plus de souplesse aux décisions qui s'appuient sur les critères suivants.

\section{CRITÈRES D'OFFICIALISATION}

Les critères présentés ici ont été élaborés au fur et à mesure des travaux de la Commission de terminologie de l'Office et des consultations qu'elle effectue périodiquement auprès des commissions ministérielles de terminologie et auprès des usagers. Ces critères tiennent compte des études et recherches effectuées sur la normalisation linguistique, et des commentaires transmis à l'Office sur l'application de son mandat de normalisation.

\section{AVIS DE NORMALISATION}

D'une façon générale, l'Office peut émettre des avis de normalisation (au sens de la Charte) dans les cas suivants :

- Remplacement de formes inappropriées ou erronées, en particulier dans l'usage institutionnel (ex. : remplacement de sole par plie [produits de la pêche]).

- La délimitation de formes concurrentes qui nuisent à l'efficacité de la communication - (ex.: timber et lumber).

- Résolution d'un problème d'ambiguïté par la définition des notions (ex.: audience et audition [justice]).

- Officialisation d'un emprunt ou d'un régionalisme (ex.: rang, en toponymie). Il est à noter dans le cas des emprunts et des québécismes, compte tenu de l'importance particulière de ces deux phénomènes, que l'Office a jugé nécessaire d'élaborer des énoncés de politique particuliers où sont examinés les cas d'acceptation, de nonintervention ou de rejet ${ }^{5}$.

- Dénomination d'une réalité nouvelle, soit qu'il n'y ait pas encore de terme français, soit qu'il y ait foisonnement de synonymes parmi lesquels il y a lieu de choisir (ex.: mesure d'atténuation [environnement]).

- Choix terminologique en vue de la modification d'un texte législatif (ex.: terminologie de la sécurité du revenu).

- Résolution d'un problème à caractère juridique (ex.: terminologie des produits de la pêche).

\section{AVIS DE RECOMMANDATION} suivants:

Les avis de recommandation sont préférés aux avis de normalisation dans les cas 
- Absence de consensus étendu. Les avis terminologiques et linguistiques sont le résultat de recherches et de consultations auprès des milieux de spécialité; l'absence de consensus du milieu joue donc un rôle essentiel dans le statut de la décision (ex.: vidéotique).

- Ouvrages terminologiques. Les ouvrages terminologiques, étudiés globalement selon des critères spécifiques touchant la méthodologie suivie, la nomenclature et la consultation, font l'objet d'avis de recommandation.

- Solution à problème strictement linguistique. Les problèmes à caractère linguistique peuvent faire l'objet d'une demande d'avis officiel (ex.: questions typographiques, orthographiques, etc.). Toutefois, bien que l'étude de ce type de dossier relève du mandat de l'Office sur l'amélioration de la langue (ex.: la féminisation des titres et de fonctions), le recours à la normalisation ne peut être, en cette matière, qu'exceptionnel.

\section{NORMALISATION ET AMÉNAGEMENT LINGUISTIQUE}

La normalisation terminologique, telle qu'elle est pratiquée par l'Office de la langue française, s'intègre dans le cadre de l'activité générale d'aménagement linguistique. Ses visées sont, en uniformisant le vocabulaire de la langue de l'Administration, d'améliorer la communication entre l'État et ses administrés.

La pratique normalisatrice de l'Office, quoique modeste, s'inscrit dans le mouvement général de la normalisation terminologique. Tant au plan national qu'au plan international, on assiste à une croissance importante du «marché» de la normalisation, conséquence de la multiplication et de la diversification de la communication spécialisée. L'importance numérique des notions à dénommer et la recherche de l'efficacité de la communication renforcent le besoin de précision en matière de terminologie.

Dans cette perspective, la raison d'être de la normalisation terminologique devient multiple. Elle doit être perçue comme:

-_ un moyen au service de l'efficacité de la communication dans l'Administration;

- un moyen de créer des consensus terminologiques notamment dans le cas où l'usage est multiple et où l'on cherche une plus grande unité;

- un moyen de moderniser la terminologie en fonction des changements technologiques, administratifs ou culturels;

_ un moyen parmi d'autres d'influencer l'usage général de la langue et sa qualité, par le biais de la pratique langagière de l'Administration qui sert alors de modèle;

- un moyen de diminuer l'insécurité linguistique causée par le processus de changement linguistique.

Cette description des fonctions de la normalisation correspond aux fonctions d'unification et de modernisation de la terminologie telles que M. Nahir les présente dans son article sur les objectifs de la planification linguistique 6 .

La normalisation terminologique, même si elle se fonde sur une large consultation des milieux intéressés, ne demeure toutefois qu'un outil au service de la communication dont l'efficacité dépend en grande partie de la volonté des usagers.

L'implantation de la terminologie officialisée doit se faire avant tout par l'utilisation de ces termes dans les textes officiels : lois, règlements, décrets, directives, etc., qui encadrent la vie de l'Administration et dont l'impact est considérable et dont l'influence sur les pratiques langagières de la population est déterminante. 


\section{NOTES}

1. ISO 1087 (1989): Vocabulaire de la terminologie.

2. Voir à ce sujet l'article d'A. Dandonneau intitulé «La diffusion de la terminologie par les textes normatifs», Actes du cinquième colloque OLF-STQ de terminologie - terminologie et communication, Montréal, Office de la langue française, 1986, pp. 205-216.

3. KOCOUREK, Rostislav (1982): La langue française de la technique et de la science, Wiesbaden, Oscar Brandstetter Verlag, p. 199.

4. Ces avis sont publiés régulièrement dans la Gazette officielle et dans le Répertoire des avis linguistiques et terminologiques, Québec, Les Publications du Québec.

5. Énoncé d'une politique relative à l'emprunt de formes linguistiques étrangères, Montréal, Office de la langue française, 1980.

Énoncé d' une politique linguistique relative aux québécismes, Montréal, Office de la langue française, 1985.

6. NAHIR, M. (1984): «Language planning goals: A classification», Language Problems and Language Planning, pp. 294-327. 